\title{
022 IMPACT OF EXTENDING SOCIAL HEALTH INSURANCE FOR THE POOR ON FACILITY-BASED DELIVERIES IN THE PHILIPPINES
}

Karlo Paolo P Paredes. Health Care Management and Policy, Graduate School of Public Health, Seoul National University, Seoul, Republic of Korea

\subsection{6/bmjopen-2015-forum2015abstracts.22}

Background Throughout the world, countries are now leading towards Universal Health Coverage -that is, to ensure everyone get access to needed health services without suffering financial hardship. Aside from the Philippines, several Asian countries have also initiated programs to extend social health insurance for the poor. Increasing access to health services is just one of the many reasons why extending insurance coverage for the poor through government subsidies is becoming a popular policy option for countries with Social Health Insurance (SHI) systems.

Objectives The primary objective of this study is to evaluate the initial impact of the 2011 reform in the Philippines which sponsored at least 5.2 million poor Filipinos to the country's National Health Insurance Program (Philhealth). Locally known as Kalusugang Pangkalahatan (KP), the reform hopes to improve maternal health outcomes, including facility-based deliveries, as a strategy to keep up with the MDG 5 targets from which the country is unlikely to achieve.

Methods The study used a double (DID) and triple differences (DDD) estimator to evaluate the impact of extending insurance for the poor. The model was fitted in a Multiple Linear Regression (MLR) and Hierarchical Linear Regression (HLR) to control for regional differences and urban/rural divide. Data used was derived from two consecutive National Demographic and Health Surveys (NDHS) in 2008 (pre-policy) and 2013 (post-policy).

Result The results suggest that the recent effort in the Philippines to extend SHI for the poor had no short-term impact on facility-delivery. Exploring factors that hinders use of facility for delivery despite the "insured" status of the poor in a bottom-up perspective should therefore be discussed for future policy development.

Conclusion Medium- and long-term evaluation is recommended for thorough policy evaluation and future health policy development. 